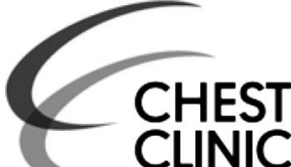

${ }^{1}$ Trauma and Emergency Surgery Unit, Department of Anesthesia and Critical Care Lyon Sud Hospital, Hospices Civils de Lyon, Pierre Benite, France

${ }^{2}$ Department of Thoracic Surgery, Louis Pradel Hospital, Hospices Civils de Lyon Bron, France

\section{Correspondence to} Dr Jean-Stephane David, Service d'Anesthésie Reanimation, Centre Hospitalier Lyon Sud, Pierre Benite, Cedex F-69495, France; js-david@univ-lyon1.fr

Received 24 December 2012 Revised 11 January 2013 Accepted 15 January 2013 Published Online First 5 February 2013

\title{
Post-traumatic pulmonary hernia
}

\author{
Jean-Stephane David, ${ }^{1}$ Candice Tassin, ${ }^{1}$ Jean-Michel Maury ${ }^{2}$
}

Post-traumatic pulmonary hernia is a very rare occurrence of chest trauma, which however remains usually covered by the skin. ${ }^{1}{ }^{2}$ A young 22-year-old male was recently admitted to our trauma centre after being thrown from a motorcycle against a road guard barrier.

Clinical examination of the patient revealed a large open wound to the upper and the anterior part of the right chest with a non-covered posttraumatic pulmonary hernia (figure 1). The CT scan of the chest (figure 2) showed the involvement of the middle and the upper pulmonary lobes in the pulmonary hernia $(*)$, a clavicle dislocation and

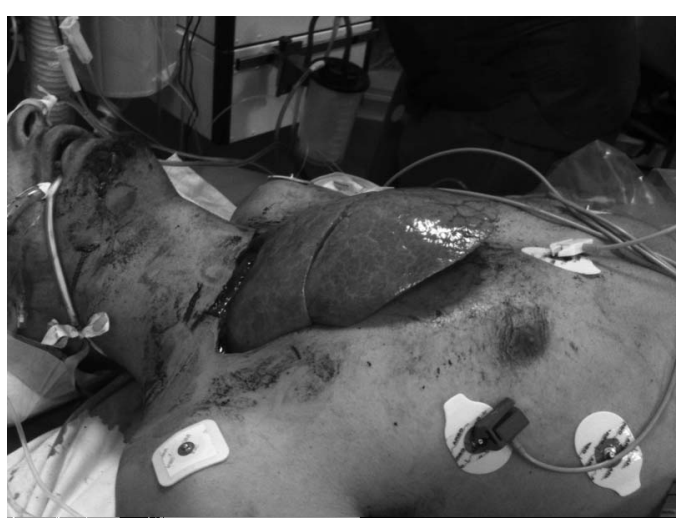

Figure 1 Pulmonary hernia observed at the admission of the patient in the trauma resuscitation unit. fractures to the first two ribs without other injury. During thoracic surgery, the lung was reintegrated to the chest cavity, the first two broken rib that were threatening the subclavian vein were resected and the proximal end of the clavicle fixed to the sternum (figure 2). Three chest tubes were inserted at the time of surgery together with broadspectrum antibiotics. Subsequently, there was no further respiratory or infectious complication. The patient was discharged from the hospital on day 10 with no further complication on day 60 .

Finally, what is sometimes surprising in traumatology is the absence of relation between the visual presentation of an injury and its severity. In other words, an injury sometimes very impressive can have a favourable outcome.

Contributors JSD, CT and JMM were in charge of the patient. JSD wrote and submitted the case. JMM and CT made corrections and gave approval for the case.

Competing interests None.

Patient consent Obtained.

Provenance and peer review Not commissioned; internally peer reviewed.

\section{REFERENCES}

1 Sarkar D, Warta M, Solomon J. Transdiaphragmatic Intercostal Herniation following Blunt Trauma. Case Rep Radiol 2012;2012:502765.

2 Saw EC, Yokoyama T, Lee BC, et al. Intercostal pulmonary hernia. Arch Surg 1976;111:548-51.

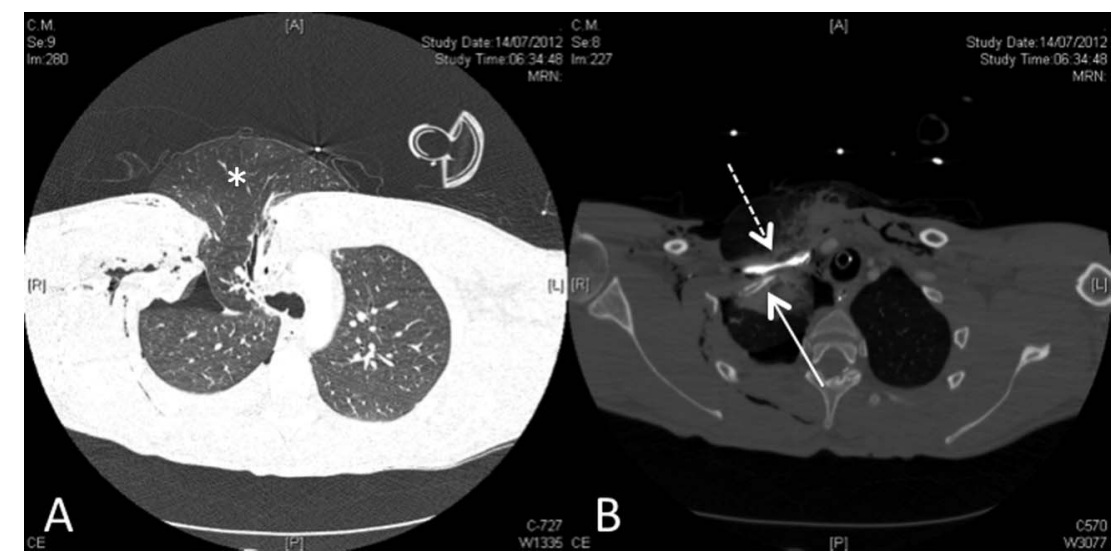

Figure 2 The CT scan of the chest showing (A) the pulmonary hernia $\left(^{*}\right)$ and (B) a broken rib (full arrow) threatening the subclavian vein (dashed arrow) after contrast injection. 